\title{
Distributed Reconstruction via Alternating Direction Method
}

\author{
Linyuan Wang, Ailong Cai, Hanming Zhang, Bin Yan, Lei Li, and Guoen Hu
}

National Digital Switching System Engineering \& Technological R\&D Center, Zhengzhou 450002, China

Correspondence should be addressed to Bin Yan; tom.yan@gmail.com

Received 25 May 2013; Revised 4 July 2013; Accepted 19 July 2013

Academic Editor: Liang Li

Copyright (C) 2013 Linyuan Wang et al. This is an open access article distributed under the Creative Commons Attribution License, which permits unrestricted use, distribution, and reproduction in any medium, provided the original work is properly cited.

\begin{abstract}
With the development of compressive sensing theory, image reconstruction from few-view projections has received considerable research attentions in the field of computed tomography (CT). Total-variation- (TV-) based CT image reconstruction has been shown to be experimentally capable of producing accurate reconstructions from sparse-view data. In this study, a distributed reconstruction algorithm based on TV minimization has been developed. This algorithm is very simple as it uses the alternating direction method. The proposed method can accelerate the alternating direction total variation minimization (ADTVM) algorithm without losing accuracy.
\end{abstract}

\section{Introduction}

Image reconstruction algorithms implemented in existing computed tomography (CT) scanners require projection data to be available in proportional space $[1,2]$. However, in CT imaging of biological specimens, data collection at a large number of projection views may result in radiationinduced object deformation. Recently, methods based on the corresponding constrained total variation (TV) or $l_{1}$-norm minimization have been widely studied in reconstruction from sparse-view data [3-9]. Sidky et al. [5] proposed the adaptive steepest descent projection onto convex sets (ASD-POCS) algorithm for CT image reconstruction. This method can realize exact-image reconstruction using fewer measurements. The alternating direction method (ADM) is an efficient approach for optimization problems. And the split Bregman-TV method based on alternating Bregman iterative approach was proposed and converged well in [8] as a solution for sparse-view CT reconstruction. A TV minimization iterative algorithm using the ADM based on augmented Lagrangian function was also proposed [10, 11]. Li et al. proposed a more robust and efficient algorithm nonmonotone alternating direction algorithm (NADA) [12] in 2012, which integrates alternating direction and nonmonotone line search. An alternating direction total variation minimization (ADTVM) algorithm for few-views reconstruction [13] was developed inspired by the literature [10-12]. The augmented Lagrangian function-based ADM is actually equivalent to the
Bregman iterative method when the constraints are linear [14]. However, the expression in [13] is simpler than that in [8].

The CT image reconstruction problem is a large-scale problem. The ADTVM algorithm [13] is not directly suitable for distributed implementation. Boyd et al. [15] argued that the alternating direction method of multipliers is well suited for distributed convex optimization, in particular, for largescale problems arising in statistics, machine learning, and other related areas. In this study, a distributed algorithm called distributed alternating direction total variation minimization (Dis-ADTVM) is developed using ADM. The proposed algorithm is as simple as the ADTVM algorithm and can accelerate the latter without accuracy loss.

\section{Method}

The imaging model can be approximated using the following discrete linear system:

$$
p=W \vec{f}
$$

where the vector $p$ has length $N_{d}$, which is the number of measured projection rays; the vector $\vec{f}$ has length $N_{i m}$, which is the number of expansion elements used in representing the object function $f(\vec{r})$; the system matrix $W$ is a pixel-driven projection operator. 


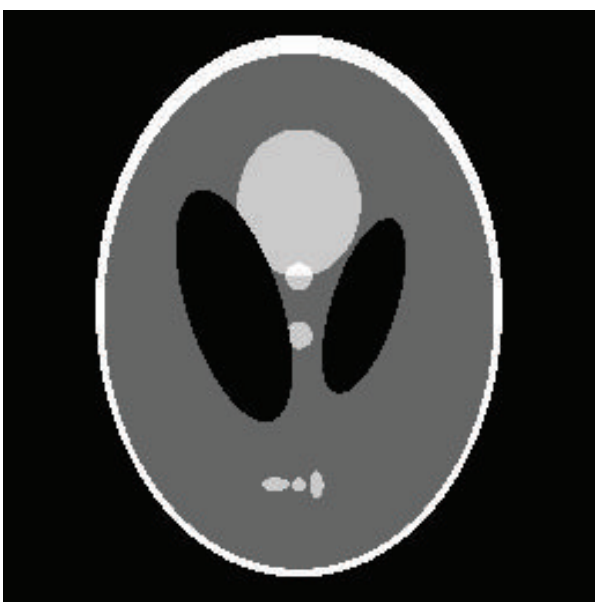

(a) Phantom

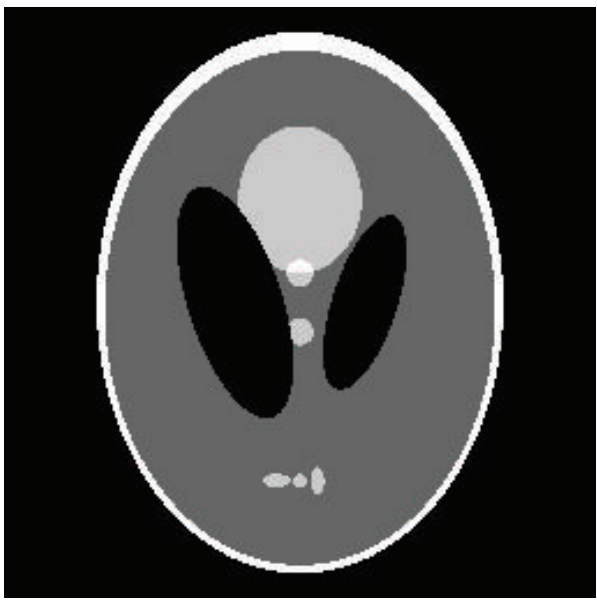

(c) ADTVM

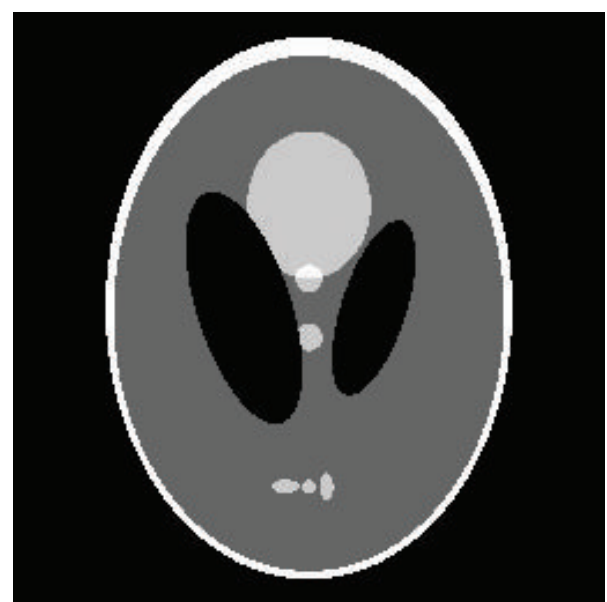

(b) ASD-POCS

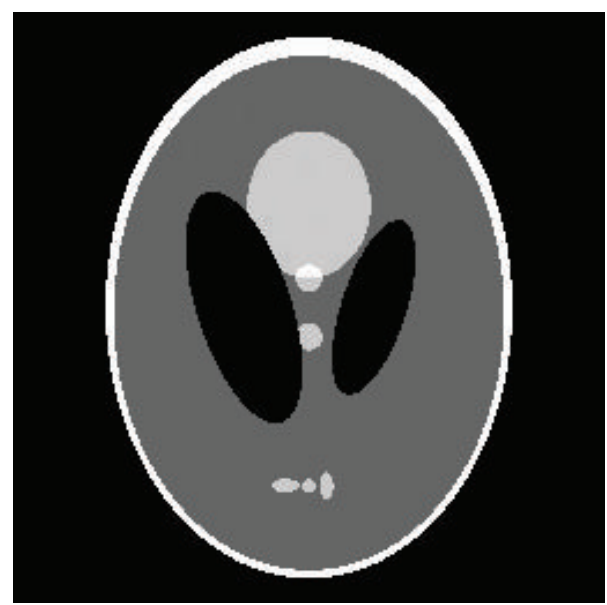

(d) New method

FIGURE 1: Image reconstruction of the Shepp-Logan phantom in a 36-view scan. Display window [0.1,0.35]. (a) Original image; (b) result of the ASD-POCS algorithm; (c) result of the ADTVM algorithm; (d) result of the proposed distributed algorithm.

Sparse-views projection data are not sufficient for exact reconstruction. The problem we consider in this study is illposed. To solve linear system (1), we use a regularization method with anisotropic TV minimization, as follows:

$$
\begin{aligned}
& \min \|\vec{f}\|_{T V} \\
& \text { s.t. } p=W \vec{f},
\end{aligned}
$$

where $\|\vec{f}\|_{T V} \triangleq \sum_{j}\left\|D_{j} \vec{f}\right\|_{1}$ and $D_{j}$ denotes the differential operator along direction $j$. In particular, $D_{1}$ and $D_{2}$ denote the horizontal and vertical differential operators, respectively, for two-dimensional form. $p$ and $W$ are separated in $i$ along vertical direction as

$$
\begin{aligned}
& \min \|\vec{f}\|_{T V} \\
& \text { s.t. } p_{i}=W_{i} \vec{f}, \quad i=1,2, \ldots, N .
\end{aligned}
$$

We consider a variant of (3) as follows:

$$
\begin{aligned}
& \min \frac{1}{2} \sum_{i}\left\|W_{i} \vec{f}_{i}-p_{i}\right\|^{2}+\lambda \sum_{j}\left\|z_{j}\right\|_{1} \\
& \text { s.t. } D_{j} \vec{f}_{i}=z_{j},
\end{aligned}
$$

where $\vec{f}_{i}$ denotes $\vec{f}$ in node $i$ and $\vec{f}_{i}=\vec{f}, i=1,2, \ldots, N$, in node $i$. Its corresponding augmented Lagrangian function is

$$
\begin{aligned}
& L_{A}\left(z_{1}, z_{2}, \vec{f}_{i}\right)=\sum_{i}\left(\frac{1}{2}\left\|W_{i} \vec{f}_{i}-p_{i}\right\|^{2}\right. \\
&+\sum_{j}\left(\lambda\left\|z_{j}\right\|_{1}+u_{i j}^{T}\left(D_{j} \vec{f}_{i}-z_{j}\right)\right. \\
&\left.\left.\quad+\frac{\rho}{2}\left\|D_{j} \vec{f}_{i}-z_{j}\right\|^{2}\right)\right),
\end{aligned}
$$

where $u_{i j}$ is Lagrange multiplier and the parameters $\lambda$ and $\rho$ are both used to balance the terms. The ADM is used to 


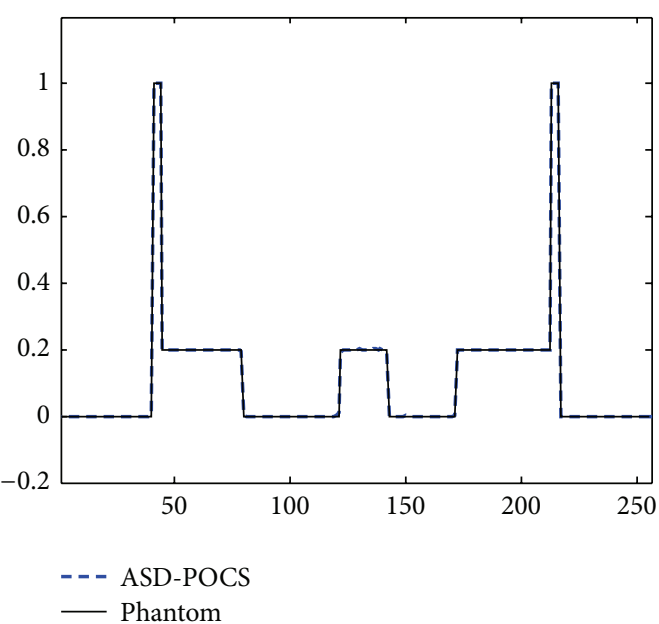

(a)

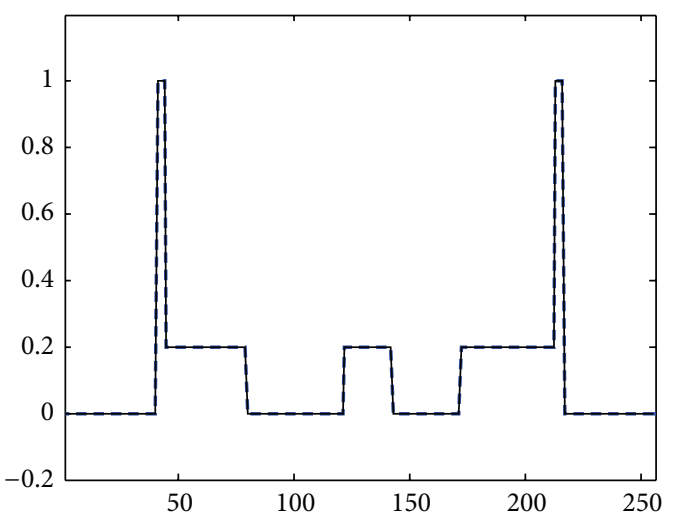

-- - ADTVM

- Phantom

(c)

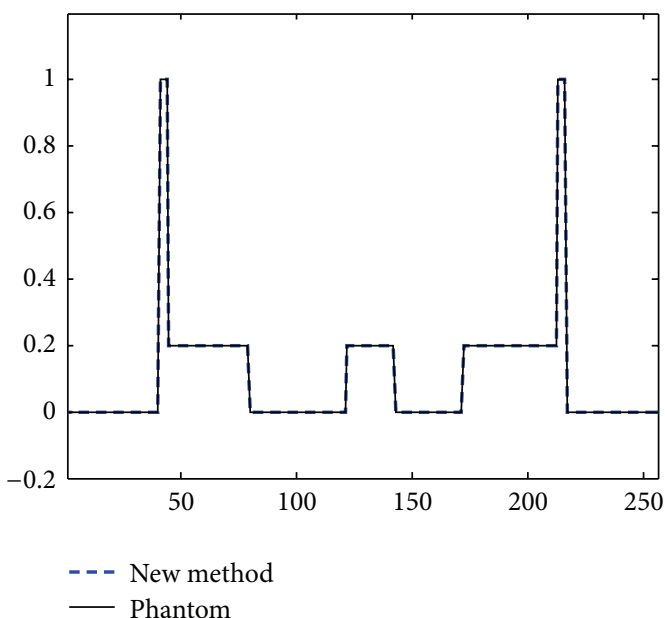

(e)

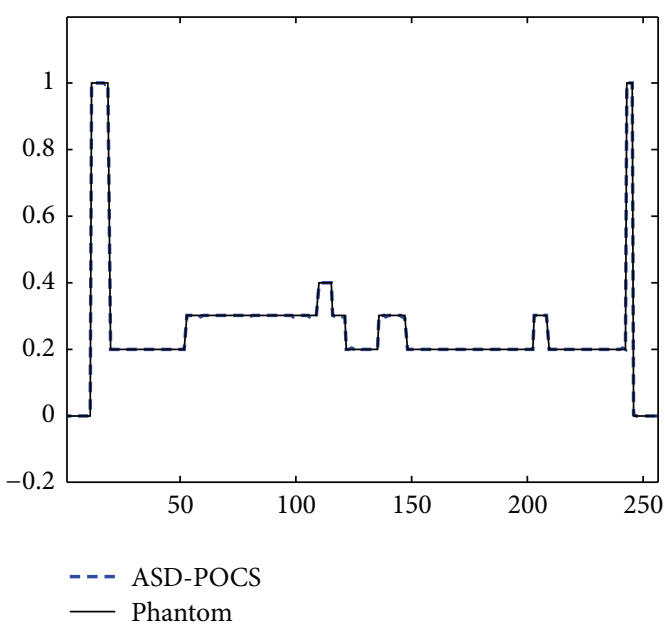

(b)

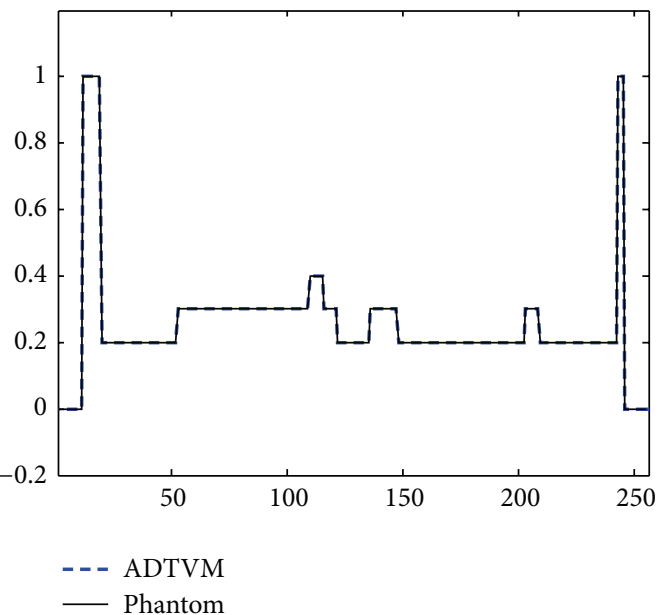

(d)

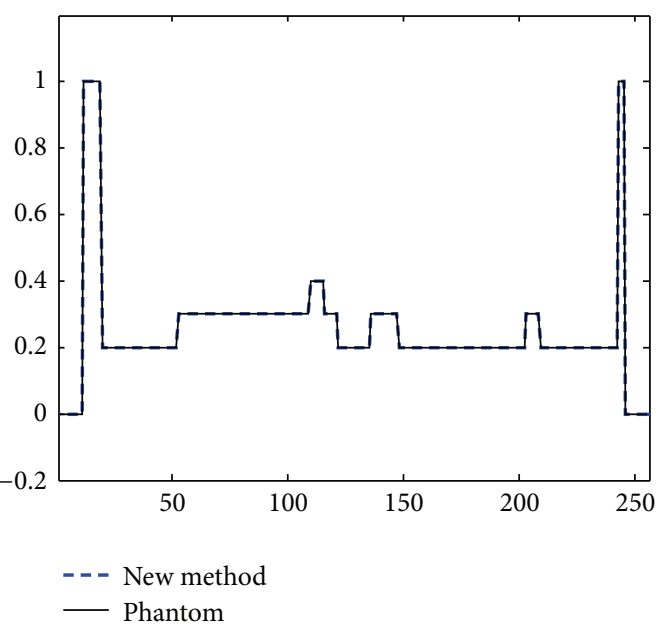

(f)

FIGURE 2: Image profiles of Figure 1. (a) Horizontal profiles along the centers of the ASD-POCS result; (b) vertical profiles along the centers of the ASD-POCS result; (c) horizontal profiles along the centers of the ADTVM result; (d) vertical profiles along the centers of the ADTVM result; (e) horizontal profiles along the centers of the proposed distributed algorithm result; (f) vertical profiles along the centers of the proposed distributed algorithm result. 


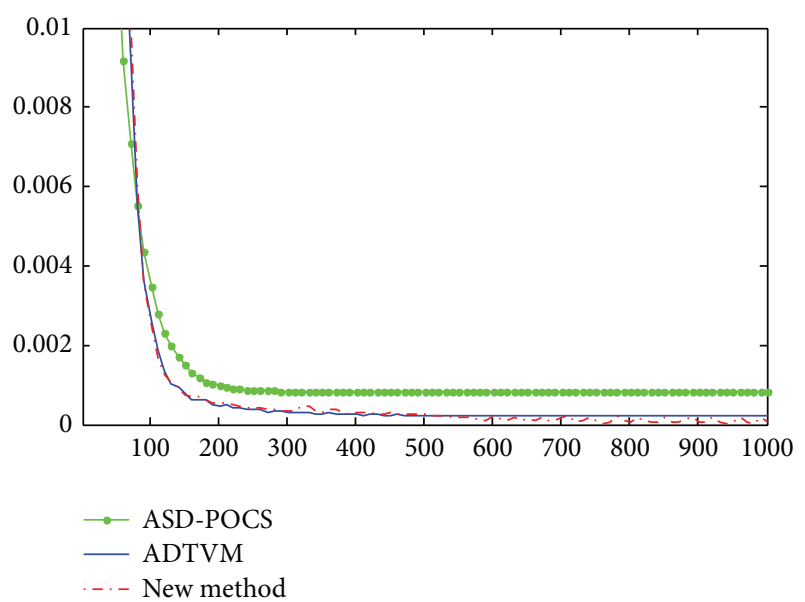

FIGURE 3: The RMSEs as functions of iterations of three tested methods.

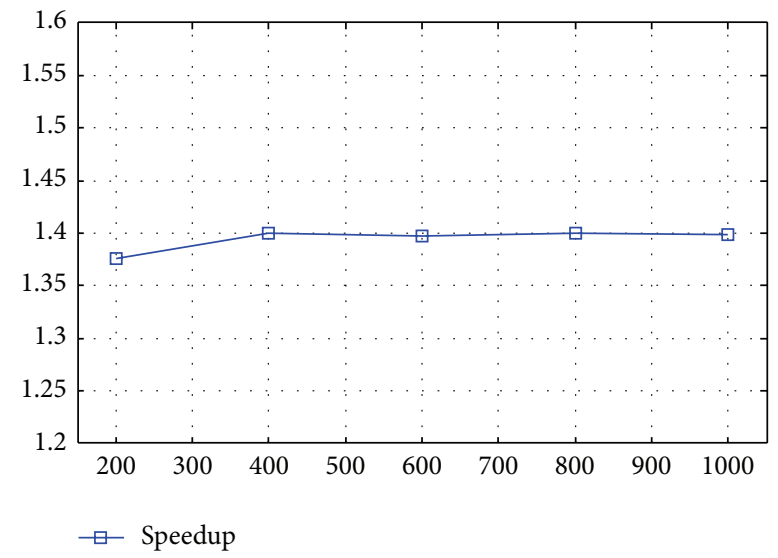

FIGURE 4: Speedup of the proposed distributed algorithm.

solve the problem that minimizes the augmented Lagrangian function; that is,

$$
\begin{aligned}
& \vec{f}_{i}^{k+1}=\underset{\vec{f}_{i}}{\operatorname{argmin}}\left(\frac{1}{2}\left\|W_{i} \vec{f}_{i}-p_{i}\right\|^{2}+\frac{\rho}{2} \sum_{j}\left\|D_{j} \vec{f}_{i}-z_{j}^{k}+u_{j i}^{k} / \rho\right\|^{2}\right), \\
& z_{j}^{k+1}=\underset{z}{\operatorname{argmin}}\left(\lambda\left\|z_{j}\right\|_{1}+\frac{N \rho}{2}\left\|D_{j} \overline{\vec{f}^{k+1}}-z_{j}+\overline{u_{j}{ }^{k}} / \rho\right\|^{2}\right), \\
& u_{j i}^{k+1}=u_{j i}^{k}+\rho\left(D_{j} \vec{f}_{i}^{k+1}-z_{j}^{k+1}\right) .
\end{aligned}
$$

The final algorithm of Dis-ADTVM can be expressed as

$$
\begin{aligned}
\vec{f}_{i}^{k+1}= & \left(\rho \sum_{j} D_{j}^{T} D_{j}+W_{i}^{T} W_{i}\right)^{+} \\
& \times\left(W_{i}^{T} p_{i}+\rho \sum_{j} D_{j}^{T}\left(z_{j}^{k}-u_{j i}^{k} / \rho\right)\right),
\end{aligned}
$$

TABLE 1: Parameters in the simulation of a sparse-view scan.

\begin{tabular}{lc}
\hline Parameters & Configuration \\
\hline Detector elements & 512 \\
Source to axis distance & $300 \mathrm{~mm}$ \\
Source to detector distance & $600 \mathrm{~mm}$ \\
Views of projection data & 36 \\
Projection data & $512 \times 36$ \\
Reconstruction size & $256 \times 256$ pixels \\
Pixel size & $0.127 \times 0.127 \mathrm{~mm}^{2}$ \\
\hline
\end{tabular}

TABLE 2: The RMSE of the three tested methods.

\begin{tabular}{lccc}
\hline & ASD-POCS & ADTVM & Distributed algorithm \\
\hline Iteration numbers & 1000 & 1000 & 1000 \\
RMSE & $8.149 E-4$ & $6.142 E-5$ & $4.777 E-5$ \\
\hline
\end{tabular}

$$
\begin{aligned}
z^{k+1}= & \max \left\{\left|D_{j} \overline{\vec{f}}^{k+1}+\frac{\overline{u_{j}^{k}}}{N \rho}\right|-\frac{\lambda}{N \rho}, 0\right\} \\
& \times \operatorname{sgn}\left(D_{j} \overline{\vec{f}}^{k+1}+\frac{\overline{u_{j}^{k}}}{N \rho}\right), \\
u_{j i}^{k+1}= & u_{j i}^{k}+\rho\left(D_{j} \vec{f}_{i}^{k+1}-z_{j}^{k+1}\right),
\end{aligned}
$$

where $\vec{f}_{i}^{k+1}$ and $u_{j i}^{k+1}$ can be computed in node $i$ and $M^{+}$ stands for the Moore-Penrose pseudoinverse of matrix $M$. Computing the pseudoinverse at each iteration is too costly to implement numerically, while we use NADA [12] to solve " $f$-subproblem" in (7).

The convergence analysis of ADTVM algorithm has been well analyzed in the literature [12], and the convergence proof of the distributed algorithms based on ADM can be found in $[15,16]$. The iterative algorithms using the ADM based on augmented Lagrangian function decompose the optimization problem into some simple subproblems with closed form solution. Therefore, the algorithms are efficient and practical for the low cost in each iteration. The NADA algorithm enables taking full advantages of the low-cost minimization in "easy" direction and allows relatively quick and large steps in the "hard" direction. The distributed algorithms can distribute some computation to individual nodes; thereby, the algorithms reduce the running time through data distribution and computation. The proposed Dis-ADTVM algorithm in this paper integrates above advantages and its derivation and implementation are as simple as the ADTVM algorithm.

\section{Numerical Results}

3.1. Simulation Studies. We perform numerical experiments to demonstrate and validate the proposed method for sparseview image reconstruction. Scanning and reconstruction parameters are listed in Table 1. Detector elements are equidistantly spaced at $0.127 \mathrm{~mm}$. 


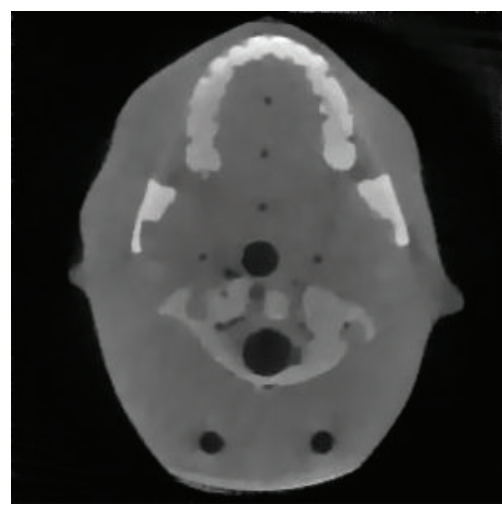

(a) ASD-POCS (200 iter)

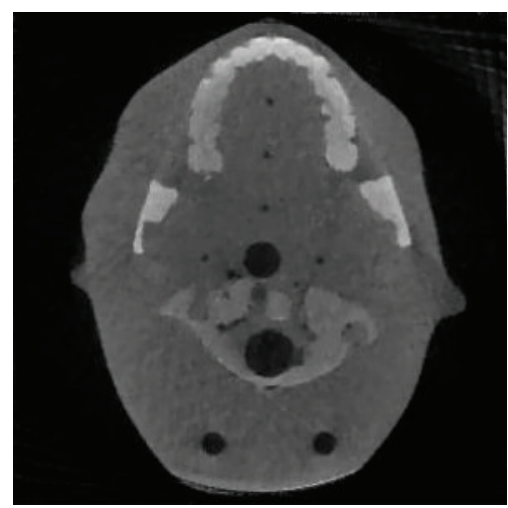

(d) ASD-POCS (1000 iter)

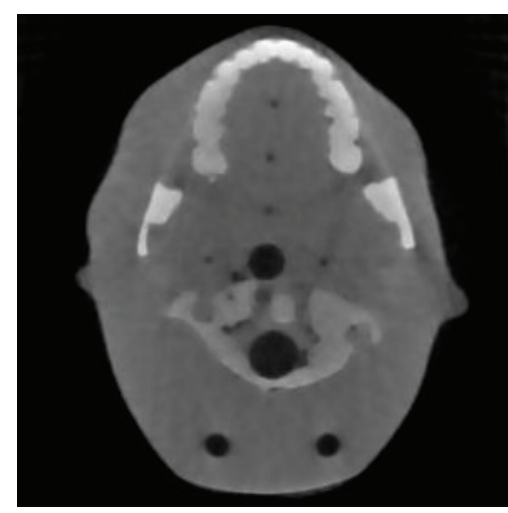

(b) $\operatorname{ADTVM}(200$ iter)

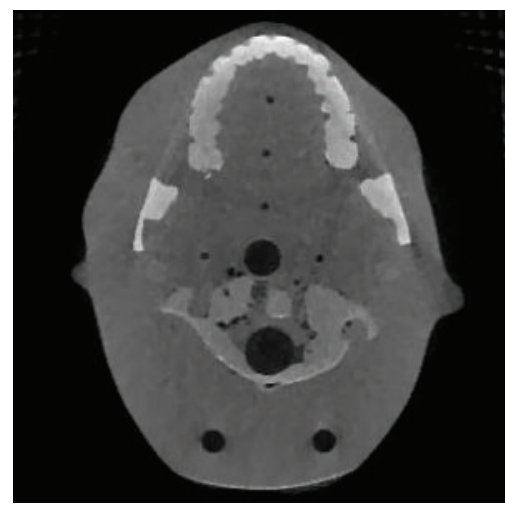

(e) ADTVM (1000 iter)

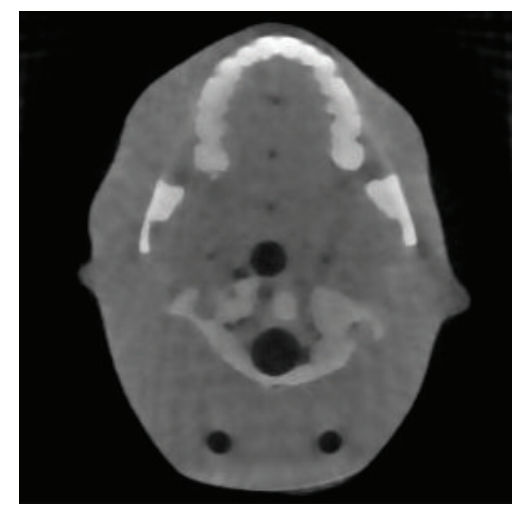

(c) New method (200 iter)

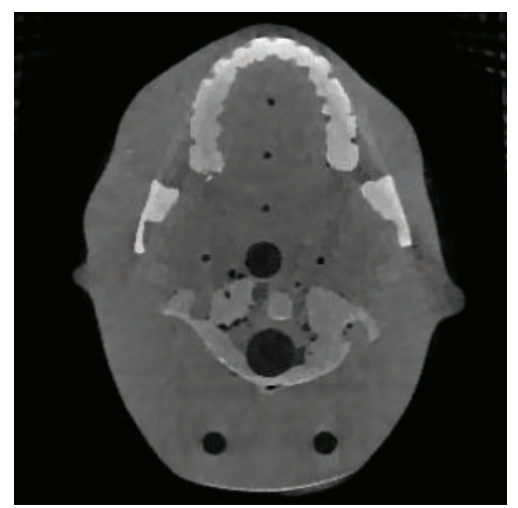

(f) New method (1000 iter)

FIgURE 5: Reconstructions of the three algorithms. (a) The ASD-POCS result with 200 iterations; (b) the ADTVM result with 200 iterations; (c) the proposed distributed algorithm result with 200 iterations; (d) the ASD-POCS result with 1000 iterations; (e) the ADTVM result with 1000 iterations; (f) the proposed distributed algorithm result with 1000 iterations.

TABLE 3: Running time of the three tested methods.

\begin{tabular}{lcccc}
\hline Iteration number & ASD-POCS $(\mathrm{s})$ & ADTVM (s) & Distributed algorithm (s) & Speedup \\
\hline 200 & 35.6462 & 24.1902 & 17.5854 & 1.3756 \\
400 & 74.8574 & 51.6406 & 36.8742 & 1.4005 \\
600 & 119.9293 & 76.7743 & 54.9518 & 1.3971 \\
800 & 145.3673 & 103.9184 & 74.2152 & 1.4002 \\
1000 & 181.7170 & 129.4053 & 92.4793 & 1.3993 \\
\hline
\end{tabular}

TABLE 4: Parameters in the real data of a sparse-view scan.

\begin{tabular}{lc}
\hline Parameters & Configuration \\
\hline Detector elements & 640 \\
Source to axis distance & $678 \mathrm{~mm}$ \\
Source to detector distance & $1610 \mathrm{~mm}$ \\
Views of projection data & 72 \\
Projection data & $600 \times 72$ \\
Reconstruction size & $300 \times 300$ pixels \\
Pixel size & $0.582 \times 0.582 \mathrm{~mm}^{2}$ \\
\hline
\end{tabular}

The proposed method is compared with ASD-POCS algorithm [6] and the ADTVM algorithm [13], using the same parameters to validate their performance. Dual core is used in implementing the proposed distributed algorithm on two nodes.

In the experimental configuration, we use one detector for data acquisition by taking 36 angular samples evenly distributed over an angular range of $360^{\circ}$. The size of the phantom simulation is set as follows. Image size is $256 \times 256=$ 65536 voxels, and projection data size is 36 views, with 512 detectors or 18432 measured rays.

The images reconstructed from this set of data using the ASD-POCS algorithm, ADTVM algorithm, and the proposed distributed algorithm are shown in Figure 1. The profiles of these images along the central horizontal and vertical rows are presented in Figure 2. The number of iterations for the three algorithms is 1000 each. The parameters of ASD-POCS are same as those in [6]. The parameters 
TABLE 5: Running time for reconstructing real data.

\begin{tabular}{lcccc}
\hline Iteration number & ASD-POCS $(\mathrm{s})$ & ADTVM (s) & Distributed algorithm (s) & Speedup \\
\hline 200 & 87.2564 & 63.1188 & 45.6657 & 1.3822 \\
400 & 184.1110 & 135.0983 & 96.6299 & 1.3981 \\
600 & 266.1320 & 214.6039 & 153.9262 & 1.3942 \\
800 & 356.8788 & 265.0989 & 190.7736 & 1.3896 \\
1000 & 445.9676 & 334.5297 & 240.6383 & 1.3902 \\
\hline
\end{tabular}

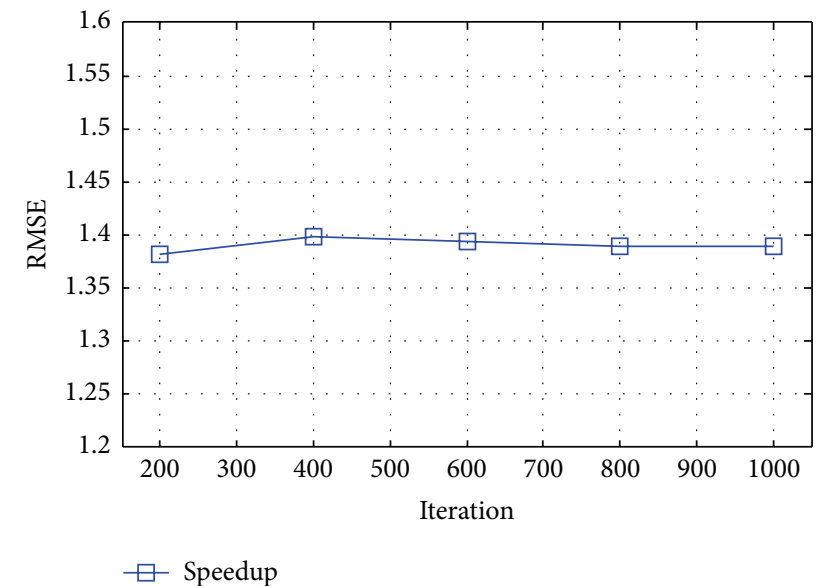

FIGURE 6: Speedup of the proposed distributed algorithm in real data reconstruction.

in the ADTVM algorithm and the proposed distributed algorithm are both $\lambda=1 / 4000$ and $\rho=32 / 4000$.

We use the root mean squared error (RMSE) as a measure of the reconstruction error to demonstrate reconstruction accuracy quantitatively. The RMSE is defined as

$$
\text { RMSE }=\sqrt{\frac{\sum_{i} \sum_{j}|f(i, j)-g(i, j)|^{2}}{N}},
$$

where $f$ and $g$ are the ideal phantom and the reconstruction, respectively, and $N$ is the total number of pixels in the image. The RMSEs of the reconstructions of the Shepp-Logan phantom are calculated. The results of the three methods are illustrated in Figure 3. Table 2 shows the RMSE of the reconstructions from the projection data above with the three algorithms. It is clear that the accuracy and effectivity of the ADTVM algorithm and the proposed distributed algorithm are both better than those of the ASD-POCS algorithm. This is due to the use of ADM and NADA algorithms. Moreover, we can see that the accuracies of the ADTVM algorithm and proposed distributed algorithm are both almost the same. This is because the derivation and implementation of distributed algorithm are very similar as the ADTVM algorithm.

The running time of the three algorithms is shown in Table 3 and Figure 4 for the phantom results in the aforementioned configuration. Timing is implemented based on the average of 10 computations. The speedup is approximately 1.4 on the average.
3.2. Reconstruction Using Real Data. We perform experiments to reconstruct a head model from real data to further validate the proposed algorithm. Scanning and reconstruction parameters are listed in Table 4. Detector elements are equidistantly spaced at $0.635 \mathrm{~mm}$.

We reconstruct a $z$-axial slice for convenience. Images reconstructed using the ASD-POCS algorithm, the ADTVM algorithm, and the proposed distributed algorithm are shown in Figure 5. The numbers of iterations for the three algorithms are 200 and 1000, respectively. The experimental result suggests that ADTVM algorithm and the proposed distributed algorithm produce better reconstruction than ASD-POCS. Hence, the results of ADTVM algorithm and the proposed distributed algorithm are almost the same.

The running time of the three algorithms is shown in Table 5 and Figure 6 for the reconstruction results in the aforementioned configuration. Timing is implemented based on the average of 10 computations. The speedup is approximately 1.4 on the average.

We use Amdahl's law [17] to predict the theoretical maximum speedup as follows:

$$
S=\frac{1}{(1-\beta) / n+\beta}
$$

where $\beta$ denotes the fraction of the algorithm which is strictly serial. In the ADTVM algorithm, $\beta$ is about 0.1 , so the theoretical maximum speedup in two nodes is $S=1.82$. The speedup in real experiments will be less than $S$ as the cost on communication exists in every iteration. The average speedup of the proposed distributed algorithm in all the experiments shows that the algorithm reduces the running time obviously.

All experiments are performed using $\mathrm{C}$ programming language under Visual Studio 2012 and OpenMP running on an AMAX Tesla workstation with Intel Xeon E5520 dual-core CPU $2.27 \mathrm{GHz}$ and $24 \mathrm{~GB}$ memories. We partly refer to the MATLAB solver of "TVAL3" [11] for the implementation.

\section{Conclusions}

The Dis-ADTVM algorithm is as simple as the ADTVM algorithm and can accelerate the latter without accuracy loss. The new algorithm is well suited for CT image sparse-view reconstruction problem as a large-scale problem. It is clear that the Dis-ADTVM algorithm can be applied to other tomographic imaging modalities with linear system models. We will study the relationship between the performance and the number of nodes in a forthcoming paper. 


\section{Acknowledgments}

This work was supported by the National High Technology Research and Development Program of China (863 Subject No. 2012AA011603) and the National Natural Science Foundation of China (no. 61372172).

\section{References}

[1] H. K. Tuy, "An inversion formula for cone-beam reconstruction," SIAM Journal on Applied Mathematics, vol. 43, no. 3, pp. 546-552, 1983.

[2] B. D. Smith, "Image reconstruction form cone-beam projections necessary and sufficient conditions and reconstruction methods," IEEE Transactions on Medical Imaging, vol. 4, no. 1, pp. 14-25, 1985.

[3] E. J. Candès, J. Romberg, and T. Tao, "Robust uncertainty principles: exact signal reconstruction from highly incomplete frequency information," IEEE Transactions on Information Theory, vol. 52, no. 2, pp. 489-509, 2006.

[4] E. J. Candès, J. K. Romberg, and T. Tao, "Stable signal recovery from incomplete and inaccurate measurements," Communications on Pure and Applied Mathematics, vol. 59, no. 8, pp. 12071223, 2006.

[5] E. Y. Sidky, C.-M. Kao, and X. Pan, "Accurate image reconstruction from few-views and limited-angle data in divergent-beam CT,' Journal of X-Ray Science and Technology, vol. 14, no. 2, pp. 119-139, 2006.

[6] E. Y. Sidky and X. Pan, "Image reconstruction in circular conebeam computed tomography by constrained, total-variation minimization," Physics in Medicine and Biology, vol. 53, no. 17, pp. 4777-4807, 2008.

[7] L.-Y. Wang, L. Li, B. Yan, C.-S. Jiang, H.-Y. Wang, and S.-L. Bao, "An algorithm for computed tomography image reconstruction from limited-view projections," Chinese Physics B, vol. 19, no. 8, Article ID 088106, 2010.

[8] B. Vandeghinste, B. Goossens, J. De Beenhouwer et al., "SplitBregman-based sparse-view CT reconstruction," in Proceedings of the 11th International Conference on Fully 3D Image Reconstruction in Radiology and Nuclear Medicine, pp. 431-434, 2011.

[9] X. Han, J. Bian, E. L. Ritman, E. Y. Sidky, and X. Pan, "Optimization-based reconstruction of sparse images from fewview projections," Physics in Medical and Biology, vol. 57, no. 16, pp. 5245-5273.

[10] Y. Wang, J. Yang W Yin, and Y. Zhang, "A new alternating minimization algorithm for total variation image reconstruction," SIAM Journal on Imaging Sciences, vol. 1, no. 3, pp. 948-951, 2008.

[11] C. Li, An Efficient Algorithm for Total Variation Regularization with Applications to the Single Pixel Camera and Compressive Sensing, Rice University, Houston, Tex, USA, 2009.

[12] C. Li W Yin, H. Jiang, and Y. Zhang, "An efficient augmented Lagrangian method with application to total variation minimization," Rice University CAAM Technical Report TR12-13, 2012.

[13] H. Zhang, L. Wang, B. Yan, L. Li, X. Xi, and L. Lu, "Image reconstruction based on total-variation minimization and alternating direction method in linear scan computed tomography," Chinese Physics B, vol. 22, no. 7, Article ID 078701, 2013.

[14] S. Osher, W. Yin, and D. Goldfarb, "Bregman iterative algorithms for 11-minimization to compressed sensing," SIAM Journal on Imaging Sciences, vol. 1, no. 1, pp. 143-168, 2008.
[15] S. Boyd, N. Parikh, E. Chu, B. Peleato, and J. Eckstein, "Distributed optimization and statistical learning via the alternating direction method of multipliers," Foundations and Trends in Machine Learning, vol. 3, no. 1, pp. 1-122, 2010.

[16] Z. Peng, M. Yan, and W. Yin, "Parallel and distributed sparse optimization,” http://www.caam.rice.edu/ optimization/disparse/.

[17] Wikipedia, “Amdahl's law," http://en.wikipedia.org/wiki/ Amdahl's_law. 


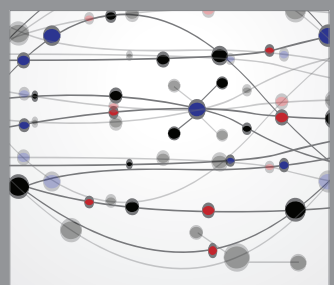

The Scientific World Journal
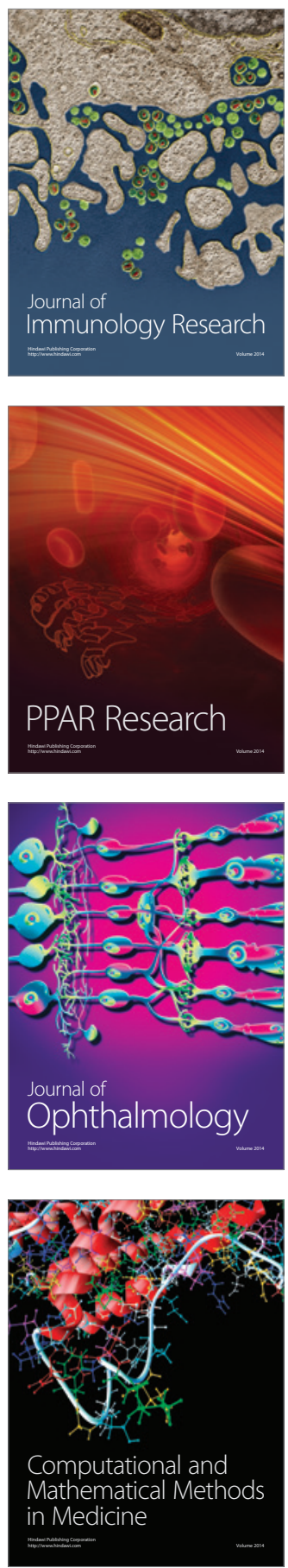

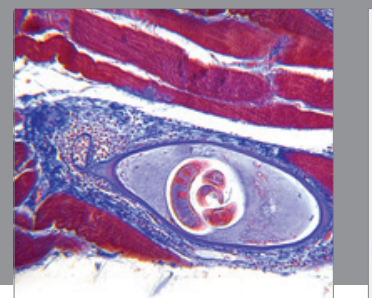

Gastroenterology

Research and Practice
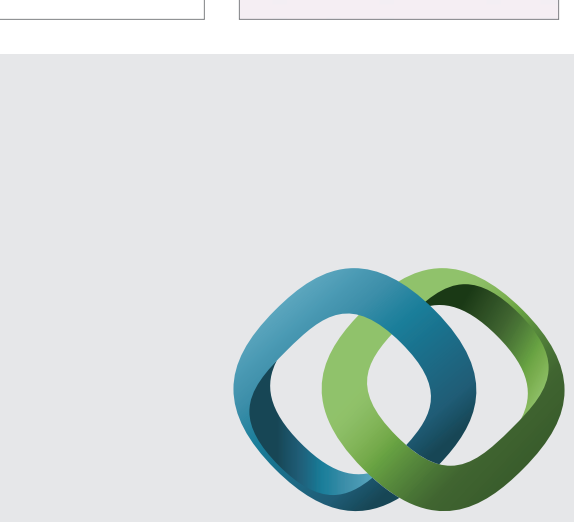

\section{Hindawi}

Submit your manuscripts at

http://www.hindawi.com
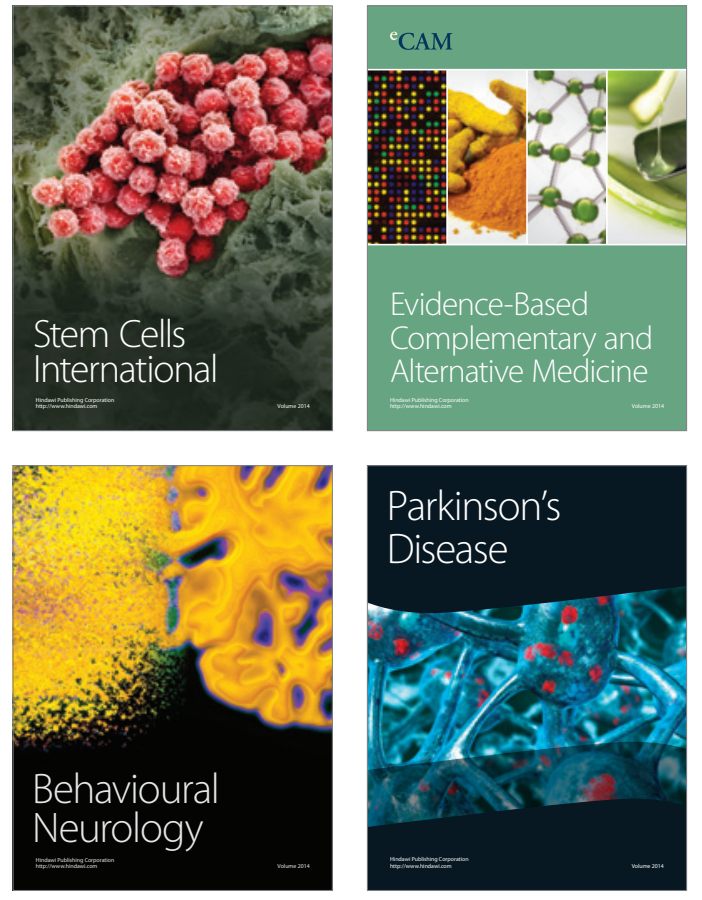
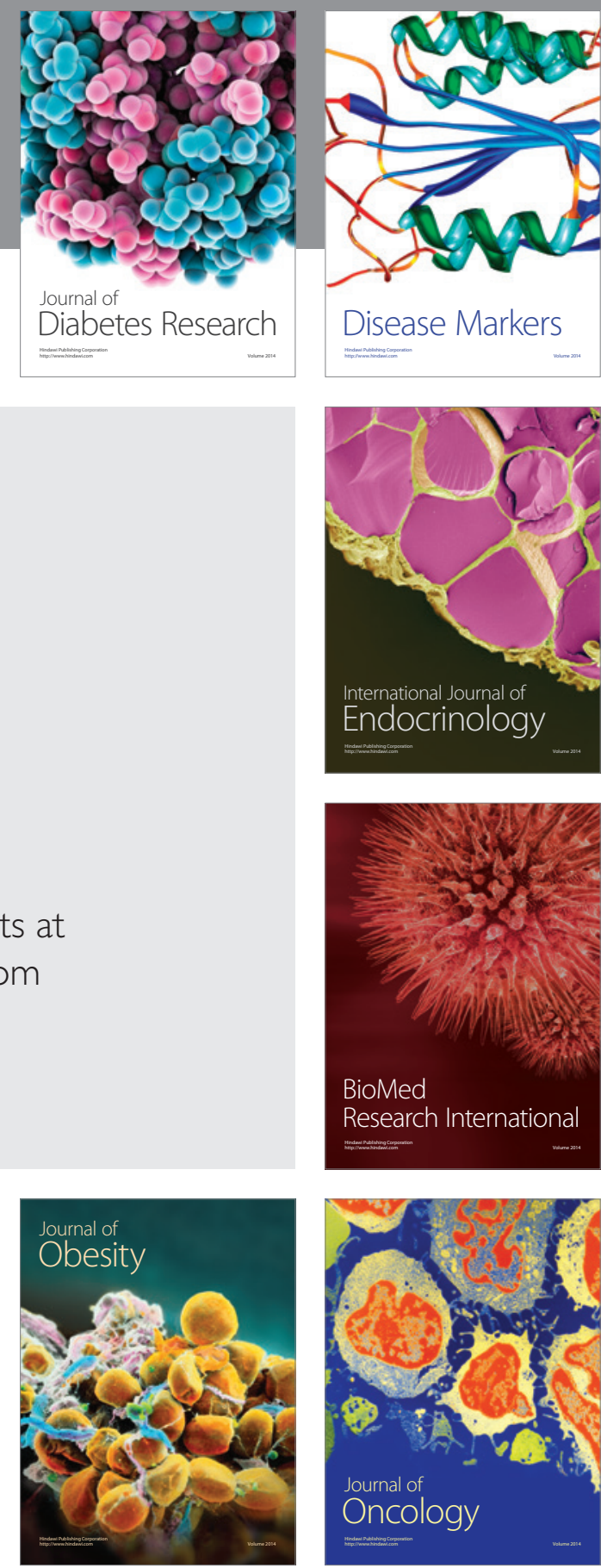

Disease Markers
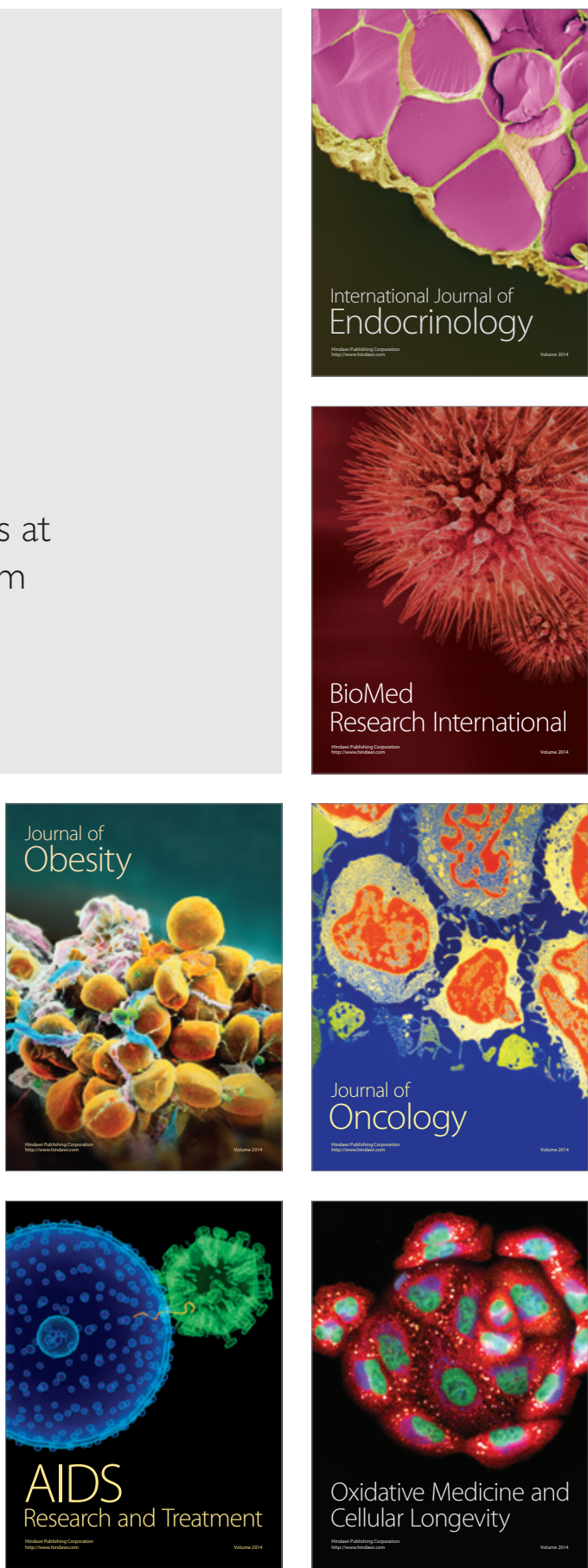